\title{
Displacement of an Electrically Charged Drop on a Vibrating Bath
}

\author{
M. Brandenbourger, N. Vandewalle, and S. Dorbolo* \\ CESAM-GRASP, Physics Department, University of Liège, B-4000 Liège, Belgium
}

(Received 1 July 2015; published 26 January 2016)

\begin{abstract}
In this work, the manipulation of an electrically charged droplet bouncing on a vertically vibrated bath is investigated. When a horizontal, uniform, and static electric field is applied to it, a motion is induced. The droplet is accelerated when the droplet is small. On the other hand, large droplets appear to move with a constant speed that depends linearly on the applied electrical field. In the latter regime, high-speed imaging of one bounce reveals that the droplet experiences an acceleration due to the electrical force during the flight and decelerates to 0 when interacting with the surface of the bath. Thus, the droplet moves with a constant average speed on a large time scale. We propose a criterion based on the force necessary to move a charged droplet at the surface of the bath to discriminate between constant speed and accelerated droplet regimes.
\end{abstract}

DOI: 10.1103/PhysRevLett.116.044501

Open microfluidics aims to transport and manipulate droplets. The transport should be quick, without alteration of the droplet content, and should allow one to perform operations including sorting, mixing, and analyzing the fluids. Open devices were demonstrated to be an interesting way to perform these different tasks, especially for microchemical analysis purposes [1]. In Ref. [2], Seo et al. created a platform that allows one to manipulate a single droplet on a flexible superhydrophobic surface. The path followed by the droplet is fully programmable. In the same spirit [3], Katsikis et al. developed a system that can manipulate numerous droplets on a surface using a magnetic field. The surface is patterned with magnetic elements that define tracks followed by the droplets in the manner of an electronic circuit. In this Letter, we show that a charge droplet can be manipulated at the surface of a vibrated bath of oil. The main advantage is that a droplet is never in contact with the surface while the programmability of the path is preserved.

Methods have been developed to study charged droplets in nonconductive liquids [4]. But, the study of a charged droplet in air has been limited to small charged droplets via electrodynamics [5] or acoustic traps [6]. We propose a method to manipulate an electrically charged droplet on a vertically vibrating bath of oil, i.e., without any contact between the liquid and the charged droplet.

The vibrating bath storage system is based on delayed coalescence. When a droplet falls on a liquid bath, the intervening air layer takes a finite time to drain from underneath the droplet. On the other hand, when the bath oscillates above a certain amplitude and frequency, the droplet may bounce and the air layer is regenerated at each bounce [7]. In the case of a high viscosity liquid bath (more than $200 \mathrm{~mm}^{2} / \mathrm{s}$ ), the bath can be considered "rigid" [8] and the droplet bounces at the same place on the bath.
We have chosen a bath of silicone oil whose cinematic viscosity is $1000 \mathrm{~mm}^{2} / \mathrm{s}$ as in Ref. [8]. The charged droplet was made of silicone oil with a smaller viscosity, i.e., $1.5 \mathrm{~mm}^{2} / \mathrm{s}$. Electrically charged droplets are created in a horizontal plane capacitor as described in Ref. [9]. In the present case, the oil being a bad electrical conductor, charge induced in the droplet depends on the purity of the liquid and on the droplet generation time. In order to ensure a minimum conductivity of the silicone oil, a small amount of ethanol (less than 1\%) was added to the liquid used to generate the electrically charged droplets. The charge of the droplets was measured before and after using a Faraday cup.

The droplet radius $r$ was tuned between 0.35 and $0.9 \mathrm{~mm}$ while the measured charge carried by the droplet was in the range between 11 and $20 \mathrm{pC}$. The characteristic discharge time can be estimated, considering the ratio between the air electrical permittivity $\varepsilon_{0}$ and conductivity of the droplet $\sigma_{d}$, to be $350 \mathrm{~s}$. Therefore, during one experimental run (5 s), the charge and the radius do not change. For our experiments, letting the charged droplet evaporate while being stored on the oscillating bath allowed one to vary the droplet radius. Given the previously calculated time for the discharge, we assume the charge is conserved while the droplet slowly evaporates.

The vertical acceleration threshold of the bath needed to observe a droplet bounce depends on the frequency and on the droplet size and viscosity [10]. We found that the threshold is not affected by the charge carried by the droplet. In the following, all the experiments were performed at a frequency $f=40 \mathrm{~Hz}$ and an acceleration of $11.7 \mathrm{~m} / \mathrm{s}^{2}$ (minimum acceleration for the bouncing of the largest considered droplets).

To apply an electric field onto the electrically charged droplet, two plane electrodes were placed on opposite sides of the bath [see the sketch in Fig. 1(a)]. The use of two large 
(a)

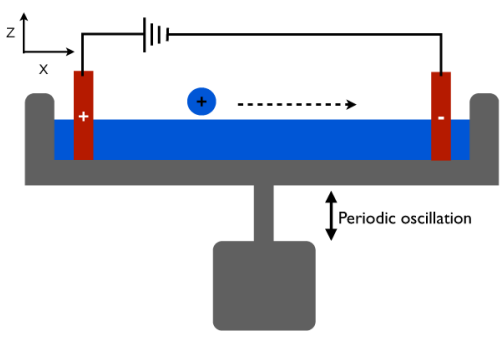

(b)

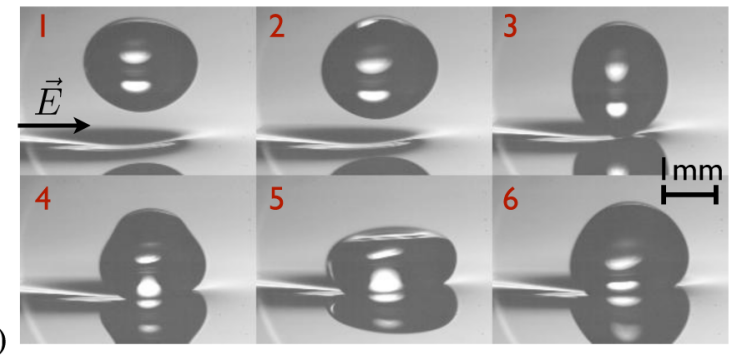

FIG. 1. (a) Sketch of the vibrating bath device (see description in the text). (b) Image sequence of the drop during one bounce. Images are spaced by $3.125 \mathrm{~ms}$. The droplet has a radius of $0.9 \mathrm{~mm}$, and the applied voltage $V$ is $5000 \mathrm{~V}$. The electrical field is oriented from left to right, and the droplet is charged positively. The bath is vibrated at $40 \mathrm{~Hz}$.

(compared to the droplet size) planar electrodes $(60 \times 45 \mathrm{~mm})$ separated by $53 \mathrm{~mm}$ allowed obtaining a homogeneous electric field between the electrodes to prevent dielectrophoresis effects.

We begin by presenting the macroscopic droplet motion, i.e., on a time scale much larger than the period of one oscillation. Then, we investigate the influence of the applied voltage and the droplet size. Finally, the mechanism of the droplet motion is determined by analyzing the trajectory of the droplet during one oscillation.

The droplet moves along the $x$ direction when a voltage is applied between both electrodes. The data of Fig. 2(a) correspond to the trajectories of the droplet taken from the experiments for which the voltage was varied while the radius was kept constant (i.e., $0.8 \mathrm{~mm}$ ). A charge $q$ of $15 \pm$ $5 \mathrm{pC}$ was measured for this set of experiments. The grey triangles, green bullets, and brown squares correspond to applied voltage $V$ of 2000, 3000, and $4000 \mathrm{~V}$, respectively. The data of Fig. 2(b) correspond to trajectories taken from experiments for which the droplet size was varied while the voltage between the electrodes $V=1000 \mathrm{~V}$ was kept constant. Open circles, triangles, and squares correspond to droplets of $0.56,0.60$, and $0.70 \mathrm{~mm}$ of radius, respectively. A charge of $14 \pm 3 \mathrm{pC}$ was measured during this set of experiments.

From these data, we deduce the following general trends: (i) large droplets $(r>0.5 \mathrm{~mm})$ have a constant speed, and this constant speed is acquired very quickly after the voltage on the lateral electrodes is switched on; (ii) for large droplets, the droplet speed increases with the applied voltage; and (iii) when the size of the droplets decreases
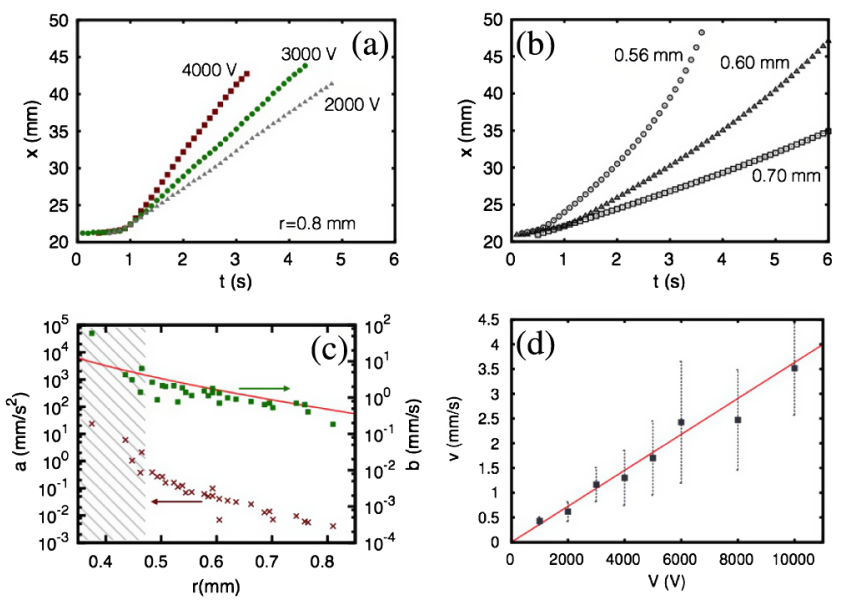

FIG. 2. (a) The trajectories $x(t)$ corresponding to charged droplets $(q=15 \mathrm{pC}$ and $r=0.8 \mathrm{~mm})$ under $2000 \mathrm{~V}$ (grey triangles), $3000 \mathrm{~V}$ (green bullets), and $4000 \mathrm{~V}$ (red squares). (b) Horizontal position $x$ over the time of $14 \mathrm{pC}$ charged droplets of radius $0.70 \mathrm{~mm}$ (open squares), $0.60 \mathrm{~mm}$ (open triangles), and $0.56 \mathrm{~mm}$ (open circles). (c) Dependence of the fitting parameters $a$ (red crosses) and $b$ (green squares) from fit with $x(t)=a t^{2}+b t+c$, as a function of the drop radius $r$. The red curve was computed according to Eq. (3). The hatching describes the crossover between the two kinds of drop motion (i.e., when the acceleration is considered negligible or not). All the experiments were made at $V=1000 \mathrm{~V}$. (d) Speed $v$ of a charged droplet $(q=11 \mathrm{pC}$ and $r=0.8 \mathrm{~mm})$ as a function of the voltage between both electrodes. The error bars are the standard deviation on ten experiments. The red line is a fit based on Eq. (3).

$(r<0.5 \mathrm{~mm})$, the second derivative of the trajectory increases. In the Supplemental Material, a movie of each behavior is shown in Ref. [11].

To determine the crossover point between the accelerated and the constant speed regimes, the trajectories of electrically charged droplets $(14 \mathrm{pC})$ were recorded for several radii during evaporation and the voltage between the electrodes was kept at $1000 \mathrm{~V}$. As a first approach, the trajectories were fitted by a parabola $x(t)=a t^{2}+b t+c$ which can be considered as the first terms of the development of any exact solution. The parameters $a$ (the second derivative) and $b$ (the slope of the trajectories) are reported as a function of the droplet radius in Fig. 2(c). The parameter $a$ (red crosses) increases when the droplet size decreases. The increase is suddenly rapid at $r \approx 0.5 \mathrm{~mm}$ showing a change of behavior for $r \approx 0.5 \mathrm{~mm}$.

In order to investigate the surprising constant speed motion which was observed in Fig. 2(a), we have studied the influence of the applied voltage on the droplet velocity. The dependence on the applied voltage for a large droplet was determined by using a droplet of $r=0.8 \mathrm{~mm}$ charged with $q=11 \pm 2 \mathrm{pC}$. The droplet speed as a function of the applied voltage between the electrodes is reported in Fig. 2(d). As seen previously [Fig. 2(a)], the higher the 
voltage, the higher the speed. The measurements show that $v$ is proportional to the voltage $V$.

To summarize, the motion of small droplets is accelerated while bigger droplets move with a constant speed. In the case of big droplets, the measurements performed at a time scale of few seconds do not present any transient regime between the droplet at rest and the constant speed regime. As soon as the voltage is switched on, the droplet moves with a constant speed. We deduce that the origin of the constant speed is to be found on the time scale of one bounce. The conclusion is that the system cannot be modeled by a charged object only influenced by an electric force and a simple law of friction. The interaction between the droplet and the bath has to be taken into account at each bounce.

The droplet motion during one bounce is therefore investigated. A high-speed camera (Phantom Miro M-310) was placed on the side of the oscillating bath in order to capture the motion of the droplet in the $x-z$ plane. The details of charged droplet $(r=0.9 \mathrm{~mm}$ and $19 \pm 5 \mathrm{pC}$ ) bounces are shown in Fig. 1(b) [11]. The voltage between both electrodes was set on $5000 \mathrm{~V}$. The displacement of the center of mass of the charged droplet is represented on Fig. 3(a). The $z$ position (vertical) and the $x$ position (horizontal) are represented by green crosses and grey circles, respectively. Along the $z$ axis, the drop bounces with a frequency of $40 \mathrm{~Hz}$, namely, the same frequency as the oscillating bath. Between two cushioned impacts with the bath, the droplet center of mass experiences successive parabolic flight in the $z$ direction. The $x(t)$ trajectory reveals a more complex structure than the macroscopic trajectory $x(t)$ presented in Fig. 2(a). Indeed, the microscopic behavior of $x(t)$ is the succession of the same pattern. Note that this kind of pattern is not observed for small droplets $(r<0.5 \mathrm{~mm})$ for which the trajectory is more complex.

To simplify, the motion of the droplet in the horizontal direction $x$ can be decomposed into two distinct steps. Step I: When the droplet is in flight, it is submitted to the

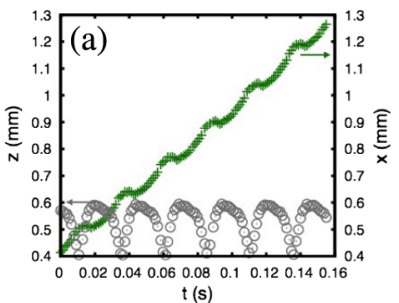

$t(s)$

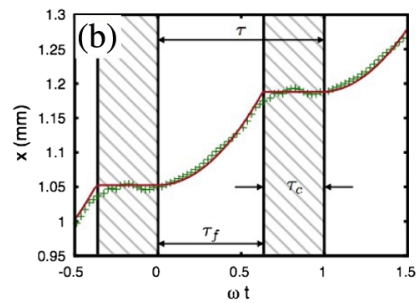

FIG. 3. (a) Horizontal and vertical positions of a charged droplet as a function of time. Green crosses and grey circles represent the vertical and the horizontal coordinates of the droplet, respectively. (b) The green crosses are the average position of the droplet over time during one bounce. The red curve is the fit made from Eq. (1) in the accelerated period (step I). The resting period (step II) is located in the shaded area. electrical force and moves in the horizontal plane, which corresponds to an accelerated movement. Step II: When the droplet interacts with the bath, the droplet has nearly no movement in the horizontal direction. As a result of steps I and II, the droplet experiences a go-stop motion. This kind of decomposition has been used to describe walkers [12] or even the skipping stone motion [13].

The horizontal motion $x(t)$ was analyzed by averaging the superposition of the periodic motion. The results are shown in Fig. 3(b) as a function of the phase $\omega t(\omega=2 \pi f)$. The droplet dynamics is then decomposed into steps I and II. To have access to the time scales of each step, we have to describe the bouncing mode of the charged droplet. As a first approximation, a bouncing droplet can be modeled by a linear spring $[8,14]$. Thanks to this hypothesis, the interaction time $\tau_{c}$ during the impact with the bath is constant and scales with the Rayleigh period [15] of vibration of a droplet, namely, $\tau_{c}=\sqrt{3 \pi m / 8 \gamma_{d}}$, where $m$ is the mass of the droplet and $\gamma_{d}$ the air-oil droplet surface tension. The interaction time is $\sim 12 \mathrm{~ms}$. This contact time is used as a characteristic time during which the charged droplet interacts with the oscillating bath.

During the "contact" time, we observed that the droplet horizontal motion is very quickly annihilated. The horizontal droplet speed is 0 during the contact time. As soon as the droplet takes off, the electrical field exerts a force on the droplet due to the excess of charges carried by the droplet. The motion can be described by considering a point charge submitted to the electric force generated by the homogeneous electric field $E=V / d$. In this case, the point charge follows the simple law

$$
x(t)=\frac{a_{c} t^{2}}{2}=\frac{q E}{2 m} t^{2}=\frac{3 q V}{8 \rho \pi r^{3} d} t^{2},
$$

where $\rho$ is the density of the droplet and $a_{c}$ is the theoretical acceleration due to the electrostatic charge. This equation was applied to the case presented in Fig. 3(b), with $d=53 \mathrm{~mm}, \rho=850 \mathrm{~kg} / \mathrm{m}^{3}$, and $r=0.9 \mathrm{~mm}$. The only parameters in the fit to Fig. 3(b) are the time of contact $\tau_{c}$ and the value of the constant in the "no flight" zone. The acceleration $a_{c}=0.34 \mathrm{~m} / \mathrm{s}^{2}$ was calculated according to Eq. (1). The result, plotted in Fig. 3(b), shows the good agreement between the theoretical trajectory and the experimental data. The deduced contact time $10 \mathrm{~ms}$ is smaller than expected (i.e., $12 \mathrm{~ms}$ ). This variation is explained by the simplifications of our model. Indeed, in the present model, the droplet deformation, the influence of the bath deformation on the droplet bouncing, the dissipation in the air film, and the characteristic time needed for the droplet to stop were not taken into account. However, in the following, we show that this simple description contains the basis and relevant physical ingredients to explain the macroscopic observations reported in Fig. 2. 
The model developed at the scale of one oscillation has to be confronted to the macroscopic behavior presented in Fig. 2. Because the drop speed is reset to 0 during one bounce, the memory of the previous jump is annealed. This statement ensures that the motion is periodic and that the average speed is constant. This later is given by the sum of the distance covered during flight time $\tau_{f}=\tau-\tau_{c}$ and during the contact time divided by the period $\tau$, namely,

$$
\langle v\rangle=\frac{q V}{2 m d} \frac{\tau_{f}^{2}}{\tau} .
$$

Equation (2) provides the dependance of $\langle v\rangle$ on the radius of the droplet $r$, namely,

$$
\langle v\rangle=\frac{3 q V}{8 \pi \rho r^{3} d}\left(\tau-\sqrt{\pi^{2} \rho r^{3} /\left(2 \gamma_{d}\right)}\right)^{2} \frac{1}{\tau} .
$$

Equation (3) was plotted in Fig. 2(c) by taking $q=14 \mathrm{pC}$, $V=1000 \mathrm{~V}, d=53 \mathrm{~mm}$, and $\gamma_{d}=0.017 \mathrm{~N} / \mathrm{m}$. The agreement between the experimental data and the model is excellent.

The relation between the average speed $\langle v\rangle$ and the applied voltage $V$ on the electrodes is also captured by Eq. (3). Indeed, Eq. (3) predicts a linear behavior of $\langle v\rangle$ with $V$ in agreement with the observations. The fit from Fig. 2(d) gives a slope of $0.36 \pm 0.02 \mu \mathrm{m} / \mathrm{s} \mathrm{V}$, which is in agreement, given the experimental error bars, with the slope computed from Eq. (2) $(0.45 \pm 0.08 \mu \mathrm{m} / \mathrm{s} \mathrm{V})$.

As indicated by the variation of the parameter $a$ of the trajectories [Fig. 2(c)], the approximation of a constant speed for the droplet is not valid for small droplets. In other words, small droplets are not stopped during the interaction with the bath. As a consequence, a criterion on the droplet size has to be provided to fit the domain of validity of our model. When the droplet falls on the oil bath, the droplet lightly bends the liquid surface. The idea is to estimate the force necessary to extract the droplet out of this dimple by exerting a horizontal force, i.e., the electrical force here. Indeed, if the electrostatic force exceeds extraction force, the droplet is not stopped when interacting with the bath.

The depth of the deformation can be approximated by balancing the kinetic energy and the energy necessary to deform the bath with a depth $h$ :

$$
\frac{2}{3} \rho \pi r^{3} v_{i}^{2}=\gamma_{b} 2 \pi r h
$$

where $v_{i}$ is the droplet speed just before interacting with the oil bath, $\gamma_{b}$ the surface tension of the oil bath, and $h$ the height of the deformation. The vertical speed $v_{i}$ is related to the flight time $\tau_{f}$, i.e., $v_{i}=g \tau_{f} / 2$, where $\tau_{f}=\tau-\tau_{c}$. This equation leads to a deformation of approximately $200 \mu \mathrm{m}$ for a millimetric droplet. This order of magnitude is in agreement with the observation (see Fig. 1 and Ref. [11]).
Let us consider that the droplet is on an incline plane when the droplet is at the bottom of the dimple. The tangent of the slope $\tan (\alpha)$ of the incline is given by the ratio $h / R$. The horizontal electric force $q E$ is not able to extract the droplet while the projection along the parallel direction of the incline of the electric force is smaller than the projection of the weight of the droplet, namely, $q E \cos (\alpha)<$ $m g \sin (\alpha)$. The inequality leads to the following criterion

$$
\frac{q E}{h r^{2}}<\frac{4}{3} \pi \rho g .
$$

Equations (5) and (4) provide the conditions on $r, E$, and $q$ to observe a constant speed motion of the droplet. In the present case, the radius $r^{\star}$ below which the electrical force $q E$ exceeds the extraction force can be computed. Taking into account the experimental conditions, namely, $q=14 \mathrm{pC}, V=1000 \mathrm{~V}$, and $d=53 \mathrm{~mm}$, one obtains $r^{\star}=0.46 \mathrm{~mm}$, which is in good agreement with the observations. A shaded area in Fig. 2(c) materializes the separation between the two kinds of motion. Droplets with size below this crossover radius are submitted to acceleration after each bounce. In this case, the main deceleration processes are dissipation in the air film during the contact time and air drag during the flight, which are far more complex to describe. Moreover, the description of the accelerated motion is more complicated because of eventual complex bouncing modes (see, for example, Ref. [16]) and implies to perform experiments with a larger oscillating bath to observe the long time scale behavior.

In conclusion, we demonstrated that charged droplets can be manipulated when they bounce on a vibrated bath. Large droplets influenced by a small electric field move with a constant speed that is proportional to the charge and the voltage. The constant speed is due to the reset to 0 of the droplet speed during each oscillation. The droplet is accelerated in the air by the electrical forces and stopped during its interaction with the oscillating bath. Small droplets influenced by a large electric field are not stopped during the contact period. Their horizontal motion is accelerated. A criterion was proposed to separate both regimes [Eq. (5)].

A complete control of a droplet on the two dimensions of the vibrating bath is presented in the Supplemental Material using crossed horizontal electric fields [11]. Experiments were also performed with success by using a water droplet and by using submerged electrodes. This system allows one to tailor or program the electrical potential landscape in which the bouncing charged droplet evolves. Finally, the criterion Eq. (5) is relevant in the study of particular bouncing droplets called walkers. Walkers are bouncing droplets that interact with the waves generated by their own impact at the surface of the bath [17]. The walker is a droplet plus the wave field and has been shown to possess some properties analogous to quantum particles [18]. Recently, Perrard et al. reported experiments concerning 
the walker motion in a harmonic well. This harmonic potential was obtained by inserting a small quantity of ferrofluid in the walker and by using a vertical external magnetic field as the center of attraction [19,20]. The interaction between an electrically charged walker and an external electrical field opens new developments in the physics of walkers.

S. D. acknowledges support as a FNRS Senior Research Associate. M. B. acknowledges support as a FNRS-FRIA Fellow. N. V. thanks the Action de Recherche Concertée (Fédération Wallonie-Bruxelles) ARC-Quandrops project. This work has been financed by the project eDroplets funded by Fonds Spéciaux pour la Recherche (FWB). This research has been also funded by the IAP 7/38 MicroMAST initiated by the Belgian Science Policy Office (BELSPO). The authors would also like to warmly thank B. Darbois-Texier, T. Truscott, N. Sampara, M. Mélard, F. Allegro, and S. Rondia for fruitful discussions and development of the experimental setup.

* Corresponding author. s.dorbolo@ulg.ac.be

[1] M. Gunji and M. Washizu, J. Phys. D 38, 2417 (2005).

[2] J. Seo, S. Lee, J. Lee, J. Seung Lee, H. Kwon, S. Cho, J. Ahn, and T. Lee, Sci. Rep. 5, 12326 (2015).

[3] G. Katsikis, J. S. Cybulski, and M. Prakash, Nat. Phys. 11, 588 (2015).

[4] W. D. Ristenpart, J. C. Bird, A. Belmonte, F. Dollar, and H. A. Stone, Nature (London) 461, 377 (2009).
[5] D. Duft, H. Lebius, B. A. Huber, C. Guet, and T. Leisner, Phys. Rev. Lett. 89, 084503 (2002).

[6] R. T. Hilger, M. S. Westphall, and M. L. Smith, Anal. Chem. 79, 6027 (2007).

[7] Y. Couder, E. Fort, C.-H. Gautier, and A. Boudaoud, Phys. Rev. Lett. 94, 177801 (2005).

[8] D. Terwagne, F. Ludewig, N. Vandewalle, and S. Dorbolo, Phys. Fluids 25, 122101 (2013).

[9] M. Brandenbourger and S. Dorbolo, J. Can. Phys. 92, 1203 (2014).

[10] T. Gilet, D. Terwagne, N. Vandewalle, and S. Dorbolo, Phys. Rev. Lett. 100, 167802 (2008).

[11] See the Supplemental Material at http://link.aps.org/ supplemental/10.1103/PhysRevLett.116.044501 for movies showing the motion of the charged bouncing droplet (high speed and low speed imaging) and the proof of concept for the manipulation of the charged droplet at the surface of the bath.

[12] C. Borghesi, J. Moukhtar, M. Labousse, A. Eddi, E. Fort, and Y. Couder, Phys. Rev. E, 90063017 (2014).

[13] J. Belden, R. C. Hurd, M. A. Jandron, A. F. Bower, and T. T. Truscott (private communication).

[14] K. Okumura, F. Chevy, D. Richard, D. Quéré, and C. Clanet, Europhys. Lett. 62, 237 (2003).

[15] Lord Rayleigh, Proc. R. Soc. London 29, 71 (1879).

[16] T. Gilet, N. Vandewalle, and S. Dorbolo, Phys. Rev. E 79, 055201 (2009).

[17] J. Molacek and J.W. Bush, J. Fluid Mech. 727, 612 (2013).

[18] J. W. Bush, Phys. Today 68, No. 8, 47 (2015).

[19] S. Perrard, M. Labousse, E. Fort, and Y. Couder, Phys. Rev. Lett. 113, 104101 (2014).

[20] S. Perrard, M. Labousse, M. Miskin, E. Fort, and Y. Couder, Nat. Commun. 5, 3219 (2014). 